\title{
Complexity-dependent modulations of beta oscillations for verbal and nonverbal movements
}

Article

Accepted Version

De Nil, L., Isabella, S., Jobst, C., Kwon, S., Mollaei, F. ORCID: https://orcid.org/0000-0003-2916-9750 and Cheyne, D. (2021) Complexity-dependent modulations of beta oscillations for verbal and nonverbal movements. Journal of Speech, Language and Hearing Research. ISSN 1558-9102 doi: https://doi.org/10.1044/2021_JSLHR-20-00275 Available at https://centaur.reading.ac.uk/97893/

It is advisable to refer to the publisher's version if you intend to cite from the work. See Guidance on citing.

To link to this article DOI: http://dx.doi.org/10.1044/2021_JSLHR-20-00275

Publisher: American Speech-Language-Hearing Association

All outputs in CentAUR are protected by Intellectual Property Rights law, including copyright law. Copyright and IPR is retained by the creators or other copyright holders. Terms and conditions for use of this material are defined in the End User Agreement. 


\section{CentAUR}

Central Archive at the University of Reading

Reading's research outputs online 
Complexity-dependent modulations of beta oscillations for verbal and non-verbal movements.

Luc De Nil, Ph.D.*,1,3, Silvia Isabella, Ph.D.*,1,2, Cecilia Jobst, M.Sc. ${ }^{2}$, Soonji Kwon, B.Sc. ${ }^{2}$, Fatemeh Mollaei, Ph.D. ${ }^{1,2}$, Douglas Cheyne, Ph.D. ${ }^{1,2}$

\section{* Co-principal authors}

${ }^{1}$ Dept. of Speech-Language Pathology, University of Toronto

${ }^{2}$ Hospital for Sick Children Research Institute

${ }^{3}$ Rehabilitation Sciences Institute

Correspondence to: Luc De Nil, Ph.D., Department of Speech-Language Pathology, Rehabilitation Sciences Building, University of Toronto, 160-500 University Avenue, Toronto, Ontario M5G1V7 (Canada)

\section{Revised manuscript submitted to}

Journal of Speech, Language and Hearing Research, November 16, 2020

Conflict of interest: there are no conflicts of interest for any of the authors on this paper.

Funding: The present study was made possible by support from the Natural Sciences and Engineering Research Council of Canada (to LDN) and the Canadian Institutes of Health Research (to DC). 


\begin{abstract}
Purpose: The planning and execution of motor behaviours require coordination of neurons that are established through synchronization of neural activity. Movement are typically preceded by event-related desynchronization (ERD) in the beta range $(15-30 \mathrm{~Hz})$ primarily localized in motor cortex, while movement onset is associated with event-related synchronization (ERS). It is hypothesized that ERD is important for movement preparation and execution, and ERS serves to inhibit movement and update the motor plan. The current study was aimed at directly comparing modulations in beta power during planning and execution of a verbal and nonverbal (button press) motor task at different levels of complexity.

Method: Seventeen right-handed adult participants ( 8 females; mean age $=25.8$ years; s.d. $=$ 5.13) completed a sequential button press and verbal task. The final analyses included data for 15 participants for the nonverbal task and 13 for the verbal task. Both tasks consisted of two complexity levels: simple and complex sequences. Magnetoencephalography was used to record modulations in beta band brain oscillations during task performance.

Results: Both the verbal and button press tasks were characterized by significant pre-movement ERD and post-movement ERS. However, only simple sequences showed a distinct transient synchronization during the pre-movement phase of the task. Differences between the two tasks were reflected in both latency and peak amplitude of ERD and ERS, as well as in lateralization of oscillations.

Conclusion: Both verbal and nonverbal movements showed a significant desynchronization of beta oscillations during the movement preparation and holding phase and a re-synchronization upon movement termination. Importantly, the pre-movement phase for simple but not complex tasks was characterized by a transient partial synchronization. In addition, the data revealed
\end{abstract}


significant differences between the two tasks in terms of lateralization of oscillatory modulations. Our findings suggest that while data from the general motor control research can inform our understanding of speech motor control, significant differences exist between the two motor systems that caution against overgeneralization of underlying neural control processes. 
The planning and execution of motor behaviour require well-tuned networks of neurons that are established through synchronization of neural activity. This synchronization is driven by oscillations, resulting from coordinated electrophysiological activity among large groups of neurons (Buzsaki, 2006; Jensen, et al., 2014). The continuum of oscillation frequencies is subdivided into various frequency bands, such as alpha $(8-12 \mathrm{~Hz})$, beta $(15-30 \mathrm{~Hz})$, and gamma $(>30 \mathrm{~Hz})$. Beta oscillations, in particular, are prominently observed in motor control (Cheyne, 2013; Khanna \& Carmena, 2015) and can be measured in motor, somatosensory and parietal cortices (Khanna \& Carmena, 2015). Magnetoencephalography (MEG), with its high temporal resolution, is a neural imaging tool well suited to the investigation of the planning and execution of motor behaviour.

Previous research on neural oscillations associated with movement control has revealed that movement execution is typically preceded by a reduction in beta power, also labelled beta eventrelated desynchronization (ERD), within the motor cortex. Movement completion, in turn, is typically characterized by event-related synchronization (ERS) to or above pre-movement levels. Gaining greater insight into the functional role of these beta responses before and during smooth and temporally-integrated motor behaviours is critical to our understanding how the brain controls and organizes sequences of movements. Different theories and hypotheses exist as to the exact functional roles of beta during movement preparation and execution. Both beta ERD and ERS have been found to be involved in serial ordering during movement planning and execution for non-speech movement task (Heinrichs-Graham \& Wilson, 2015; Tzagarakis et al., 2010). Ng et al. (2013) showed that anticipation of upcoming motor demand, which they operationalized as movement inhibition prior to uploading weight, produced pre-movement beta ERD while unexpected uploading of weight did not. Beta ERD begins prior to movement onset and reaches 
peak amplitude during movement execution, believed to be reflective of various processes during movement preparation and execution. It has been proposed that the magnitude of ERD is linked with various movement and task-related parameters, including the timing of response selection (Kaiser, et al. 2001), response preparation (Kaiser, et al. 2003), response speed (Pastotter, et al. 2012), and commission of response errors (Cheyne, et al. 2012). Little et al. (2019) speculated that beta ERS, observed following movement termination, allows for sensorimotor integration, which is congruent with the hypothesis advanced by Jenson et al. (2020) that beta ERS reflects sensorimotor integration based on available information from sensory areas.

A number of studies have observed a transient beta rebound prior to movement onset during experimentally induced hold phases. Park et al. (2013) proposed that such partial rebound may reflect intentional action inhibition prior to the go-cue that would trigger movement onset. It has been proposed that such beta rebound is modulated for different types of action sequences and may reflect differences in task complexity and demands. For instance, Jenson et al. (2020) have proposed that beta transient rebound prior to movement execution may be modulated by attentional demands. For speech, such modulations based on anticipated task demands are in line with the State Feedback Control model of speech production (Houde \& Nagarajan, 2011) and may account for the role of beta ERD in the preparatory forward modeling based on sensory predictions of the upcoming motor plan. Some indirect evidence of the relationship between motor planning and beta modulation comes from a study in children with cerebral palsy who were found to have increased beta ERD related to difficulties with anticipated grip force, thought to be associated with deficits in motor planning (Kurz et al. 2014). A number of researchers have proposed that beta modulation may reflect the updating of upcoming action goals based on 
previous inputs and observed errors (Gould et al., 2012; Tan et al., 2016; Tzagarakis et al., 2010).

Because more complex actions, typically associated with higher attentional demands, may result in more frequent error feedback, and the need of additional resources for the integration of sensory and motor outcomes, it can be hypothesized that observed amplitude and timing variations in beta modulation could be linked in part to differences in movement complexity. Research into the relationship between beta oscillations and movement complexity so far has yielded conflicting results. While one study has found increased beta ERD amplitude across four different finger sequences of increasing length and complexity (4 to 16 digits long; Manganotti, et al. 1998), most studies have reported increased spatial extent of beta ERD for complex movements, but no differences in power amplitude (Bai, et al. 2005; Chen et al. 1997; HeinrichsGraham, et al. 2015; Nakagawa, et al. 2011). Investigating whether ERD may differentiate simple from complex movements during the pre-movement planning stage, Heinrichs-Graham, et al. (2015) did not observe any such pre-movement differences for complex and simple button press sequences. In contrast, Tzagarakis et al. (2010) reported that pre-movement beta ERD amplitude was sensitive to the amount of directional uncertainty, with differences in beta ERD observed approximately $400-1000 \mathrm{msec}$ after cue onset. This suggests that the movementpreparation time-period of $500 \mathrm{msec}$ used by Heinrichs-Graham et al. (2015) may have been too short to observe differences in beta oscillatory power. Thus, it remains possible that premovement beta ERD may reflect motor complexity but only after the initial planning stages have been completed. As a result, the functional role of modulations in pre-movement beta ERD, including beta transient rebound, during movements of varying complexity, remains to be determined. 
Speech, which requires high spatiotemporal accuracy of planned speech sounds produced at a fast speed, with adult speakers usually producing up to six to nine syllables per second (Kent, 2000), places high demands on processes underlying motor behaviour. Therefore, a neural system that is capable of coordinating the planning and temporally precise release of each motor plan is critical to fluent speech production. While the importance of beta oscillatory modulations in limb and other motor behaviour has been extensively investigated, the extent to which these findings can be extended to speech motor behaviour is less well understood, although there is some evidence for similar oscillatory dynamics associated with speech production. In a review of the MEG literature, Munding, et al. (2016) reported that speech is associated with a broad bihemispheric network that, despite significant temporal overlap, shows early activation in the occipital lobe (during picture naming tasks) followed by activation in temporal, sensorimotor and inferior frontal cortex, respectively. Jenson, et al. (2014) reported alpha/beta ERD starting as early as $500 \mathrm{~ms}$ prior to speech execution, an effect that was stronger in overt compared to covert speech production. Gehrig et al. (2012) observed distinct beta desynchronization (ERD) during a sentence-reading task. The desynchronization started approximately $350 \mathrm{~ms}$ after cue presentation and was primarily left lateralized. Similar to what has been reported for other motor behaviours, beta desynchronization continued during speech motor execution, followed by a rebound in beta power (ERS) following the completion of speech. When participants were asked to deliberately withhold planned speech, Piai et al. (2015), observed a decrease in alpha-beta power over the occipital cortex during the withholding phase of the experiment, with a simultaneous increase in power over frontal regions during the same task phase. In line with previous interpretations (Park, et al. 2013), they proposed that this frontal power increase may reflect the need to inhibit 
speech onset and maintain the motor and/or cognitive state when temporarily withholding speech production.

At present, important questions remain regarding the processes driving beta oscillations, in terms of both amplitude and latency, as movements of different complexity progress from preparation to execution. In this respect, comparing speech and non-speech movements may help our understanding to what extent the observed neural modulations are task specific, or rather reflect more general motor control processes. Some have argued that speech relies on a highly specialized and distinct neural network (Ziegler \& Ackerman 2013), while others have proposed a more integrated view in which speech is controlled by a sensorimotor network that at least partially overlaps with that driving other oral or nonoral motor behaviours (Ballard, et al, 2003; Maas, 2017, Loh, et al. 2020). While speech undoubtedly has unique characteristics, such as the need for very fast and sequential coordination of respiration, phonation and articulation, each involving many muscle groups, it does share characteristics with other motor behaviour. For one, many of the neural systems that are involved in speech also have an important role in the planning and execution of non-speech motor behaviour (Loh, et al. 2020). In addition, oral structures involved in speech are essential for non-speech oral movements, such as swallowing. As such, it could be argued that significant overlap in control processes may result in a more efficient neural system. To that end, the objective of this study was to determine how verbal and non-verbal movement-related oscillatory brain patterns may be differentially affected by task complexity. We hypothesized that the observed oscillatory dynamics would be similar for the verbal and non-verbal tasks, with a distinct beta desynchronization prior to movement onset, followed by a significant re-synchronization following movement termination, but that the two tasks would differ in cortical lateralization of oscillations. Furthermore, based on prior studies on 
the role of beta ERD and ERS in non-verbal movement complexity, we hypothesized that movement complexity would affect the latency and magnitude of observed beta ERD and ERS.

\section{Materials and Methods}

\section{Participants}

Seventeen healthy adults between 19 and 35 years of age ( 9 females, 8 males; mean $=25.8$ years; s.d. $=5.13)$ were recruited for this study. While all speakers used English as their main language of communication, four participants were non-native speakers of English. Data from one female participant was excluded due to make-up induced artifacts time-locked to speech production. Data from three more participants were excluded, two due to muscle artifacts within the gamma band that impacted beta analysis and one due to technical difficulties during data acquisition. The gamma band interference was observed in two participants during data analysis and consisted of large gamma activity of unknown origin. The strength of this gamma activity partially concealed lower oscillatory frequency. In total, 15 participants ( 8 female) were analyzed for the button task, and 13 participants ( 6 female) were analyzed for the verbal task. All participants were right-handed as determined with the Edinburgh Handedness Inventory (Oldfield, 1971), had normal self-reported speech, language and hearing with no developmental or neurological history, and used English as their current primary language for at least the last 8 years. Participants practiced the pronunciation of each target syllable sequences prior to entering the scanner room. The study was approved by the Hospital for Sick Children Research Ethics Board and the Research Ethics Board at the University of Toronto, and all participants gave their informed consent. 
Stimuli and Tasks

\section{Insert Figure 1 about here}

Participants performed two tasks, a repeated verbal motor task and a repeated button press task. The order of verbal and non-verbal tasks were counterbalance across participants. Task completion took approximately 20 minutes per participant. For each task, blocks of six pseudorandomly selected simple and complex response trials were interleaved for a total of 240 simple and 240 complex trials. Each block was pseudo-randomized so that simple trials were followed by complex trials $50 \%$ of the time, and vice versa. All trials consisted of a sequence (simple or complex) that was shown on a computer screen for $800 \mathrm{~ms}$, followed by a randomized inter-stimulus interval of $800-1800 \mathrm{~ms}$ ("hold phase") after which a green square ("GO") cue appeared for 500ms. Participants were instructed to wait for the cue before producing the presented button press or verbal task. They had a $1600 \mathrm{~ms}$ window to complete the response before the next trial appeared. In both tasks, participants were instructed to respond as quickly and accurately as possible, but not to correct themselves. Each block of trials was about 6 minutes long, with 20 seconds between blocks and 5 minutes between speech and button press tasks.

Each sequence consisted of a series of 4 responses. For the verbal task, three verbal utterances $(/ \mathrm{pa} /, / \mathrm{ta} / \mathrm{and} / \mathrm{ka} /)$ were selected that represented separate articulatory motions. Specifically, /pa/ involved bilabial lip closure and opening, /ta/ involved alveolar tongue tip movement, and $/ \mathrm{ka} /$ involved tongue dorsum movement. All consonants were voiceless to facilitate acoustic analysis (see below). In the simple trials, participants produced a series of 4 identical utterances (e.g., /pa pa pa pa/). For the complex trials, a pseudo-random sequence of the four syllables was presented (e.g., /pa ta ka pa/). Across sequences, the first syllable was evenly utilized (i.e., there were the same number of trials that started with $/ \mathrm{pa} /, / \mathrm{ta} /$ or $/ \mathrm{ka} /$ ). The following 
sequences included each of the remaining two syllable options followed by a randomly selected repeat. Participants completed the verbal task using their natural speech pattern.

For the button press task, participants rested their hand on a response box with three buttons. Each button was associated with a number ("1", "2" or "3"), which corresponded to a specific finger ("1"=index finger, "2"=middle finger and "3"=ring finger). Sequences were created similar to the verbal task, with simple sequences consisting of 4 identical numbers (e.g., $/ 1111$ /) and complex sequences containing all three digits followed by one randomly selected repeat (e.g., /1-3-2-3/). Only the right (dominant) hand was tested and no specific instructions were given regarding timing pattern.

\section{Data acquisition}

All participants were seated upright during the experiment on a padded chair within the MEG chamber. Neuromagnetic brain activity was recorded using a whole head 151-channel Magnetoencephalography (MEG) system (CTF MISL, Coquitlam, BC, Canada) at the Hospital for Sick Children (Toronto). Data were collected at 1,200 samples/s (0-300Hz bandpass filtered) for the button press task, and at 12,000 samples/s $(0-3000 \mathrm{~Hz}$ bandpass filtered) for the verbal task. The verbal task was sampled at a higher rate to preserve the speech signal quality collected within the MEG dataset. Button press responses were collected within the MEG dataset using a nonmagnetic fiber optic response pad (LUMItouch Response System, Lightwave Medical Industries, Burnaby, Canada). Head position was continuously monitored with three fiducial coils placed at the left and right pre-auricular regions and one on the bridge of the nose (nasion). These coils allowed for the measurement of head movement during the scan as well as to align localized MEG response sources onto the subject's own anatomical Magnetic Resonance Image (MRI). T1- 
weighted structural MR images were obtained on the same day from each subject using a 3T Siemens Magnetom Trio scanner (gradient echo sequence (flipangle D 9_, TE/TR D 2.96 ms/2300 ms, 192 sagittal slices, $1 \mathrm{~mm}$ thick, $256 \_256$ matrix, 25.6cm FOV) at the Hospital for Sick Children.

\section{Movement Onset and Offset Identification}

In order to determine pre-motor response patterns, the response onset for each sequenced trial had to be identified. For the button press task, this was done using unique binary codes associated with each response button recorded by the MEG acquisition system. An off-line MATLAB script was used to identify the onset of the first and last responses for each trial. For the verbal task, time-frequency spectrograms of the acoustic signal were generated to identify the onset of /p/, /t/ and /k/ consonants based on the acoustic burst in the spectrogram (Kent \& Read, 1992). Customized MATLAB software was used to identify the precise onset latencies of the acoustic bursts. Simple and complex trials were labeled, and marker times were saved into the continuous MEG datasets for further analyses. In order to determine the reliability of these measures, an independently trained rater remeasured 60 trials each of 4 randomly selected subjects. Histogram analyses revealed that $77.9 \%$ of inter-reliability markings were within 0.005 seconds of the original rater, while intra-reliability scores showed $90.24 \%$ accuracy. None of these differences were statistically significant.

Movement offset for the button press task was determined based on the timing of the last button press. For the verbal task, movement offset could not be identified reliably from the recorded acoustic signal associated with the final vowel. Therefore, task offset was approximated by identifying the onset of the vowel in the last syllable of the four-syllable sequence, analogous to the timing of the last finger press in the nonverbal task. Using a custom-written script in 
MATLAB (The MathWorks, Inc) the first formant frequency of the last vowel was identified for simple and complex trials using linear predictive coding (LPC) in a series of overlapping 20-ms windows of speech signals incremented every $4 \mathrm{msec}$. This allowed us to identify the onset of the vowel automatically, check for errors, and align the markers with the MEG data.

\section{Data analysis}

For each task, continuously recorded MEG data were segmented into 10-second time windows for response-locked onsets (6 seconds pre and 4 seconds post "first verbal onset" and "first button press") as well as for cue and stimulus onsets ( 4 seconds pre and 6 seconds post "cuestimulus-locked" and "stimulus-onset-locked) datasets. All epoched datasets were down-sampled to $600 \mathrm{samples} / \mathrm{s}$ and filtered at 1-150 Hz. Any trials that exceeded a mean sensor position of $1 \mathrm{~cm}$ or more away from the mean head position or had sensor activity exceeding 5 picoTesla (generally as the result of eye blinks or muscle artifacts) were excluded from further analysis.

Group-averaged source analyses co-registered each participant's own MRI with MEG source localizations and normalized into standard adult MNI template space using SPM8 (Welcome Institute of Cognitive Neurology, London, UK). Localization of significant brain activity was carried out using both event-related (Cheyne et al., 2006, 2007) and frequency-based (Robinson and Vrba, 1998) beamformer algorithms utilizing covariance weighted time windows surrounding time-locked button press and verbal responses, and restrictions to the outer surface of the brain using a single-sphere head model (Lalancette et al. 2011).

Beta band $(15-30 \mathrm{~Hz})$ frequency changes of induced cortical oscillations were measured using a Synthetic Aperture Magnetometry (SAM) algorithm (Robinson \& Vrba, 1998) with a 4mm resolution, implemented in the BrainWave MATLAB toolbox (Jobst et al. 2018; 
cheynelab.utoronto.ca/brainwave). Whole-brain noise normalized difference (pseudo-T) images were calculated using a $400 \mathrm{~ms}$ baseline from the dataset epoched to stimulus onset ( 0 to $400 \mathrm{~ms}$ post stimulus onset) that was subtracted from $400 \mathrm{~ms}$ windows of the dataset epoched to the first response from 0 to $1600 \mathrm{~ms}$ (post first button press or first plosive). Images were thresholded using a paired random permutation test (2048 permutations) for each condition (e.g., simple vs. complex), and Bonferroni corrected for multiple comparisons $(p<0.05)$ and then scanned to identify the largest magnitude changes within the sensorimotor cortex regions. These peak locations (in Talairach coordinates) were used for the calculation of detailed frequency fluctuations over time (in addition to changes in signal strength), called time-frequency representations (TFR), where wideband $(1-30 \mathrm{~Hz})$ and narrowband $(15-30 \mathrm{~Hz})$ source activity were measured and analyzed. The peak images were also interpolated onto high-resolution cortical surfaces, extracted from an individual's own MRIs using the CIVET pipeline (Kim et al. 2015), for a threedimensional visualization of the brain sources found from each task.

Statistical analysis

To compare task-induced source activity across trial types, time-frequency representations (TFRs) were constructed from the peak source locations determined from the pseudo-T images. This was accomplished using a Morlet wavelet frequency transformation (Tallon-Baudry et al., 1997) using 9 cycles per wavelet over the frequency range of $15-30 \mathrm{~Hz}$ in $1 \mathrm{~Hz}$ steps. All waveforms were compared statistically with a method described by Guthrie and Buchwald (1991) that estimates significance of consecutive paired t-tests between two time series and corrected for multiple comparisons. Peak latency and amplitude for beta ERD and ERS, were analyzed using a $2 \times 2$ within-subject ANOVA comparing sequence complexity across hemispheres for the button press and verbal tasks. 


\section{Results}

All subjects complied with task instructions and completed the tasks. Trials were excluded from analysis due to performance errors (premature or late responses), technical issues or inability to place reliable measurement markers ( simple button press $=0.8 \%$, complex button press $=0.8 \%$, simple verbal $=6.0 \%$, complex verbal $=7.4 \%$.

\section{Button Press Task}

All subjects performed the button press task with pseudo-randomly interleaved simple and complex stimulus sequences. As can be seen in Table 1, mean reaction time (RT) was faster $(p<$ .001 ; paired t-test) for simple sequences than for complex sequences; $p<.001$; paired t-test). Mean response duration (RD) was not significantly different between simple sequences and complex $(p=.19 ;$ paired t-test).

\section{Insert Figure 2 and $\underline{\text { Table } 1}$ about here}

Next, virtual sensors were plotted within the left and right hemispheres at the source location for datasets epoched to the three time points of interest: stimulus onset, first response onset and last response onset (Figure 2). Time courses of neuromagnetic activity in the beta frequency band accompanying simple and complex sequences were compared using a paired random permutation test. Descriptively, stimulus onset resulted in an initial decrease in beta power (ERD). For simple, but not complex, sequences this was followed by a transient partial synchronization. Subsequently, both simple and complex stimuli resulted in further decreases in beta power prior to the onset of the first button press. 
The latency of ERD onset was similar across sequence types and hemispheres $\left(\mathrm{F}_{\text {sequence }}(1,14)=0.3, p=0.58 ; \mathrm{F}_{\text {hemisphere }}(1,14)=2.0, p=0.18\right)$. In contrast, the amplitude of the ERD peak revealed a main effect of hemisphere but not sequence type $\left(\mathrm{F}_{\text {sequence }}(1,14)=3.1, p=\right.$ $\left.0.10 ; \mathrm{F}_{\text {hemisphere }}(1,14)=80.9, p<0.001\right)$, revealing a greater $\mathrm{ERD}$ in the left compared to the right hemisphere. Following the initial peak ERD, the differences in ERD magnitude within the left hemisphere between simple and complex sequences reached statistical significance $(p<0.05)$ for the time interval from 0.61 seconds post-stimulus until 0.07 seconds prior to the onset of the first button press. Similarly, there were statistically significant differences within the right sensorimotor cortex from 0.7 seconds post-stimulus until 1.01 seconds prior to onset of the first button press.

Following the last button press, ERS was observed starting a few hundred milliseconds after the response and reaching a peak prior to the onset of the following trial (see Table 2 for means and standard deviations). There were no significant difference in ERS peak latency found between sequences or hemispheres $\left(\mathrm{F}_{\text {sequence }}(1,14)=0.2, p=0.66\right.$; $\mathrm{F}_{\text {hemisphere }}(1,14)=0.5, p=$ 0.50). The amplitude of the ERS peak was significantly stronger for the complex than the simple sequence in the left compared to the right hemisphere $\left(\mathrm{F}_{\text {sequence }}(1,14)=40.1, p<0.001\right.$; $\left.F_{\text {hemisphere }}(1,14)=16.4, p=0.001\right)$. Statistically significant differences in the magnitude of beta ERS between simple and complex tasks continued within the left sensorimotor cortex from 0.8 seconds after the last button press until onset of the subsequent trial, and in the right sensorimotor cortex from 0.3 seconds after the last button press until the onset of the subsequent trial.

Verbal Motor Task 
As described in the method section, the speech analysis was based on data from 13 participants. As shown in Table 1, mean reaction time was observed to be faster for simple sequences than for complex sequences $(p<.0001)$. Mean response duration was shorter for simple sequences than for complex $(p=0.02)$.

\section{Insert Figure 3 about here}

In order to determine the relationship between verbal sequence complexity and beta oscillations, neuromagnetic activity was analysed similar to the button press task. Data were epoched to stimulus onset to examine preparatory activity, to onset of the first plosive to examine activity related to response execution, and to onset of the last formant to examine beta ERS. Source activity was localized using SAM beamformer analysis, revealing beta band desynchronization in bilateral sensorimotor cortices (Figure 3). For simple and complex sequences epoched to stimulus onset, activity was localized to the left and right precentral, postcentral, and inferior frontal gyri (Brodmann Areas 6 and 9; see Table 3 for coordinates). Data epoched to the first plosive and to the last formant also showed significant activation in Brodmann Areas 6 and 9, with additional peak activation in the primary sensorimotor cortex (BA 3) and Insula (BA 13).

\section{Insert $\underline{\text { Table } 3}$ about here}

Virtual sensors were plotted at the source location for datasets epoched to the three time points (Figure 4). Time courses of neuromagnetic activity in the beta frequency band accompanying simple and complex verbal sequences (Figure 5) were compared using a paired random permutation test, revealing a bilateral pattern of activity similar to that observed during the button press task.

\section{Insert Figure 4 and Figure 5 about here}


The latency of ERD onset following stimulus presentation was significantly slower in the right compared to the left hemisphere, but no effect of sequence was found $\left(\mathrm{F}_{\text {sequence }}(1,12)=\right.$ $\left.0.48, p=0.50 ; \mathrm{F}_{\text {hemisphere }}(1,12)=9.4, p=0.01 ;\right)$. A similar pattern of effects was found for the amplitude of the ERD peak, which was significantly greater in the left than the right hemisphere, without an effect of sequence type $\left(\mathrm{F}_{\text {sequence }}(1,12)=2.9, p=0.12 ; \mathrm{F}_{\text {hemisphere }}(1,12)=8.1, p=\right.$ 0.015). Following peak ERD, significant differences were observed between simple and complex verbal sequences within the left sensorimotor cortex from 0.67 seconds post-stimulus until 1.03 seconds prior to the onset of the first syllable (all $p<0.05$ ). Similarly, there were significant differences in the right sensorimotor cortex from 0.68 seconds to 0.79 seconds post-stimulus and again from 0.93 seconds to 1.0 seconds post-stimulus. Regarding the post-movement ERS following the last syllable, there were no significant differences in ERS peak latency $\left(\mathrm{F}_{\text {sequence }}(1,12)=1.13, p=0.31 ; \mathrm{F}_{\text {hemisphere }}(1,12)=0.25, p=0.63\right)$, or peak amplitude $\left(\mathrm{F}_{\text {sequence }}(1,12)=3.15, p=0.10 ; \mathrm{F}_{\text {hemisphere }}(1,12)=0.28, p=0.61\right)$. However, prior to the ERS peak, differences in ERS amplitude between sequence types were found within the left sensorimotor cortex from 0.26 seconds to 0.41 seconds after the last formant and again from 0.56 seconds to 0.75 seconds after the last formant. No significant ERS differences were found within the right sensorimotor cortex. See Table 2. For mean and +/- standard error results.

\section{Insert Table 2 about here}

\section{Discussion}

The main objectives of the research study were to investigate modulations in beta oscillations during the planning and execution of a verbal task, to compare these modulation 
patterns with those observed during a non-verbal (button press) task, and to investigate the influence of task complexity on these oscillatory modulations.

As expected, based on previous studies (Christina, et al. 1982; Fischman, 1984; Klapp, 2003), simple sequences in both the verbal and button press task yielded significantly faster movement initiation times compared to the more complex sequences. On the other hand, mean sequence duration was significantly longer for the complex compared to the simple sequences, but only for the verbal task. This was somewhat surprising as it was expected that mean duration would be longer for complex sequences in both tasks as the execution of button press sequences involving different fingers could be expected to take longer than a sequence involving movements of a single finger. One possible explanation is that the verbal task was inherently more complex than the button press task, and therefore had a higher load on the motor planning and execution system especially when different articulators were involved. This possibility finds some indirect support in the observation that both the initiation and duration times for the verbal sequences were longer than for the nonverbal sequences. Indeed, mean duration time for the simple verbal sequence was descriptively longer than that observed for the complex nonverbal sequence. Alternatively, because mean nonverbal sequence duration was shorter than for the verbal task, it may be that the lack of a statistically significant difference reflected a floor effect whereby it becomes increasingly more difficult to produce a sequence faster. Further research will be needed to confirm these findings.

During the button press task, ERD was observed following stimulus onset bilaterally in the primary motor and sensorimotor cortex corresponding to areas associated with hand movements (Mayka, et al. 2006; Cheyne, et al. 2006). Peak ERD was found to be significantly stronger in the left compared to the right hemisphere. A similar pattern was observed for the 
verbal task with beta desynchronization observed bilaterally in premotor and motor cortical areas typically associated with articulatory movements, but statistically stronger in the left hemisphere (Figure 4). The laterality effect is not surprising given that all participants completed the nonverbal task with their right (dominant) hand, and the left hemisphere is typically the dominant hemisphere for speech in right-handed individuals. In addition, the verbal task was associated with a shorter ERD peak latency in the left hemisphere. ERD latency has been suggested to be a measure of the onset of motor preparation (Tzagarakis, et al. 2010) and may be related to the complexity of cognitive processes involved in the selection of a motor response (Kaiser, et al. 2001). This could help explain the shorter latency in the left hemisphere, which is the dominant processing site for speech-related motor tasks, such as the verbal task used in this study. The fact that ERD latency differences were observed for the verbal but not the non-verbal task provides partial support to our interpretation the two tasks may have differed in inherent complexity (see above). In contrast to the button press task, the verbal task was also associated with additional modulations in sensorimotor cortex and insula. While there has been some debate regarding the role of insula in speech and nonspeech tasks (Dronkers, 1996), our current findings of increased modulation at the level of the insula, together with the observation of complexitydependent timing differences between the verbal and nonverbal tasks, support the hypothesis that the insula may have a stronger functional role in speech than nonspeech task, especially when the two tasks differ in their attentional demands (Eckert, et al. 2009).

The initial peak ERD did not differentiate significantly between simple and complex sequences, in contrast to some previous studies that reported significant differences based on movement complexity (Manganotti, et al. 1998; Chen, et al. 1997; Nakagawa, et al. 2011). However, studies have defined complexity in various ways. For instance, complexity in the study 
by Manganotti, et al. was defined as the number of elements in the sequence (finger-thumb opposition task), ranging from 4 to 16 finger movements in a sequence. In the study by Nakagawa, et al. (2011), complexity was defined by the use of the dominant versus the nondominant hand during the completion of finger tapping and chopstick handling tasks. The lack of task-related differences for peak ERD in our study may suggest that the two tasks were similar in the timing of motor planning initiation and putting the motor system in a general state of motor readiness (Cheyne, 2013).

An important observation in the current study was that both the verbal and nonverbal tasks showed a transient partial resynchronization during the movement planning, beginning after peak ERD. However, this partial resynchronization was only present during the simple sequence task. In other words, after the initial ERD reached its peak, beta power remained stable for complex sequences, but showed a transient reduction in ERD for simple sequences (see Figure 3A-B). A similar transient synchronization was observed in the study by Tzagarakis, et al. (2010) during tasks with varying levels of movement uncertainty. Park, et al (2013) also reported the occurrence of transient resynchronization during simple repetitive arm movements, but not during more complex movement sequences. This transient resynchronization appears to be specific to the delay period between stimulus and response onsets, given that shorter delays to response do not show the same phenomenon (Heinrichs-Graham, et al. 2016). With regard to speech, the presence of transient partial re-synchronization for simple repetitive syllable sequences but not for the more complex non-repetitive sequences may reflect the role of various motor planning processes prior to speech. Sternberg, et al. (1980) proposed that the speech planning phase is initiated by the initial detection of the signal followed by the decision to respond. Subsequently, appropriate motor subprogram(s) are identified and located, a process 
that may be affected by the number of units in a sequence. This is followed by the initiation of the command sequence and the time needed to execute the components of the motor program. Within this model, it can be hypothesized that the initial ERD peak observed in our study corresponds to the initial decision to respond following stimulus detection and the general readiness for planning and executing the motor task. In the case of a repetitive simple verbal task, only one subprogram associated with the CV syllable needs to be identified and located. At that point, the motor system must be inhibited in order to prevent the initiation of motor execution until the go-signal is given. This pre-motor inhibition is reflected in a transient partial resynchronization (Walsh, et al. 2010). Following the go-signal, ERD power increases again in order to allow the execution of the motor sequence. In contrast, for the complex verbal sequences, multiple motor subprograms, associated with different $\mathrm{CV}$ syllables, need to be identified, located and unpacked prior to movement execution. As such, there may be less of a need to inhibit the premature start of motor execution and as a result no transient resynchronization is needed.

The modulation pattern differences observed between simple and complex sequences may also reflect the differential role of the phonological loop during speech preparation. According to Guenther (2016), the phonological loop consists of two components: the articulatory loop and the phonological store. The articulatory loop allows for covert rehearsal of the sequence, while the phonological store maintains the covert responses in preparation for overt execution. Within this model, peak ERD can be hypothesized to reflect the early motor programming and unpacking of motor plans associated with the stimulus sequence. Simple repetitive sequences are easier to store in memory and may not trigger extensive pre-articulatory rehearsal. As a result, ERD desynchronization is reduced and the partial resynchronization again 
reflects the inhibition of motor execution. In contrast, the more complex sequences trigger more extensive pre-articulatory rehearsal and associated activation of the phonological store, demonstrated by the maintenance of ERD rather than a partial resynchronization. These two hypotheses do not need to be mutually exclusive and further research will need to determine the differential role of these various pre-articulatory processes during speech. For instance, it might be possible that a longer hold phase would reveal partial resychronization even for the more complex sequences, once the need for a pre-articulatory rehearsal process has been reduced.

An alternative explanation for the more pronounced ERD amplitude observed in the complex condition could be the involvement of a greater number of effectors (fingers or articulators) during that sequential tasks. However, such an interpretation does not seem to be supported by the findings by Park, et al. (2013), who reported that the number of effectors was not related to magnitude of ERD during a 1- or 2-finger button response task. Similarly, Isabella et al. (2015) did not find any beta ERD differences between preparing two responses (index and middle finger) versus one (index only) during a button response task. Therefore, it seems unlikely that the current findings can be explained based on differences in number effectors used during the task, and most likely reflects differences in task complexity. While this observation appears to contradict an earlier report by Heinrichs-Graham and colleagues that complexity did not affect beta ERD (Heinrichs-Graham, et al. 2015), the difference between that study and our own may be explained by the presence of a longer delay period between stimulus presentation and movement initiation in our experimental design, as discussed above. Importantly, the observed ERD differences between simple and complex tasks were present during the planning stage following the initial ERD peak, and not during the execution phase. Therefore, it is clear 
that complexity-dependent modulations of beta oscillations are not related to response execution, but rather are reflective of a 'hold' period prior to movement execution.

Significant ERD differences between the simple and complex verbal tasks in our study, timed with the transient partial synchronization, occurred at approximately $600 \mathrm{msec}$ post stimulus onset for both the left and right hemisphere. This observation supports the interpretation that the observed synchronization reflects the need for greater inhibition of the simple verbal sequence response during the planning phase at a time when the motor system is ready for movement initiation. Based on a review the MEG data for speech, Munding, et al. (2016) reported that the average word production latency is around 600 to 700 msec. Similarly, Hasan, et al. (2015) reported that the brain networks involved in phonetic and articulatory movement initiation typically became activated around 481-535 milliseconds following stimulus presentation, although they also noted significant interspeaker variability at this stage in speech production. In our study, the difference between simple and complex became statistically significant after the onset of the transient partial synchronization following the peak ERD, suggesting that the actual synchronization started a bit earlier than when significance was reached, which would mean that our data is largely in congruence with the timing reported in these previous studies.

A noticeable difference between our findings for the verbal and the button press task was that beta ERD during movement planning was less pronounced for the verbal than for the button press task, especially in the left hemisphere. In addition, while the transient partial synchronization for the simple sequence resulted in a reduced ERD especially in the left hemisphere in the planning phase for both of our tasks, the difference remained throughout most of the planning phase for the button press task, while the amplitude of the beta oscillations 
during the verbal task desynchronized again more quickly to a level similar to that observed for the more complex sequence. One reason for the reduced ERD for the verbal compared to the button press task may be that the sequential verbal task, which involved phonotactically permissible consonant-vowel combinations in English, represent a more frequently used response for our participants compared to the less familiar button press task (Maassen, personal communication; Tremblay, et al. 2007), resulting in a reduced ERD and thus less of a need for a strong movement inhibition. The fact that the ERD onset for the verbal sequences occurred sooner in the left hemisphere after stimulus onset than it did for button press also may suggest that the verbal task was cognitively less complex than the nonverbal task. One could argue that this interpretation does not seem to be supported by the lack of a significant complexity effect for peak ERD in either task in our study. However, it is possible that the complexity difference between verbal and button press is greater than that between our simple and complex sequences. On the other hand, our behavioural data showing increased reaction and duration times for verbal than nonverbal tasks may not support the hypothesis of a reduced processing load for the verbal tasks. Further research is needed to examine the effects of task complexity, possibly by comparing beta with alpha ERD responses, as the latter are thought to more closely reflect attentional demands and cognitive effort, which would be directly correlated with task complexity (Wostmann, et al. 2017).

In addition to a complexity-dependent transient partial synchronization prior to movement, our data showed that movement termination resulted in a complexity-dependent effect on ERS. Event-related synchronization is typically seen following movement offset and is thought to reflect cortical motor inhibition due to movement-generated sensory activity (Gaetz, et al. 2010, Cheyne, 2013). Post-movement beta synchronization also may reflect tracking of 
motor outcome and sensory cues used in updating existing motor programs (Little, et al. 2019; Mary, et al. 2015). For both tasks, there was a left-lateralized stronger beta ERS for complex compared to simple sequences. Event-related synchronization is known to be modulated by a number of different variables, including errors (Tan, et al. 2014), practice (Ricci, et al. 2019), and by forced termination of movements (Alegre, et al. 2008). The fact that the observed ERS was stronger for complex than for simple sequences suggests that cortical motor inhibition of the ipsilateral sensorimotor cortex following cessation of the movement is stronger for complex than for simple responses. Moreover, it is possible that the complex movement sequences, involving multiple effectors, resulted in more pronounced sensory feedback. Given that ERS is involved in sensorimotor integration (Tan, et al. 2016) and may reflect updating of the motor plan based on sensory feedback, the differences in amplitude of the modulation may reflect this updating process. In other words, there may be a greater need to update the motor plan based on sensory feedback for complex over simple sequences, which is reflected in our data as greater ERS amplitude.

There are a number of limitations in the present study that require further exploration in follow-up studies. First, it is possible that our chosen manipulation of task complexity did not sufficiently differentiate between simple versus complex movements and that a more pronounced differentiation may have yielded more significant results between the two levels. Secondly, the complex trials in the current study also involved multiple effectors (fingers or oral articulators) compared to the single effector in the single trial. While it can be argued that the use of multiple effectors represents an increase in movement complexity, it would be useful in future studies to consider the differential effects of motor versus cognitive task complexity. Thirdly, production errors during the task were not considered as part of our data analysis. Our analysis was 
primarily focused on the planning phase prior to motor execution and as such errors during movement execution were not part of the analysis, nor was our study design aimed at maximizing the likelihood of errors. Such an analysis would provide valuable additional insights, especially if the number of errors could be increased by stronger manipulations of levels of task complexity. Fourthly, the verbal and non-verbal task were relatively artificial in that they did not consider higher level cognitive factors that have an important role during more typical speech and motor tasks. It would be of significant interest in future studies to expand the experimental design with verbal tasks that manipulate higher level cognitive-linguistic processes, as well as more goal-oriented nonverbal tasks. Finally, while all of our speakers used English as their main language of communication, 4 of our participants were non-native speakers of English. While we believe that the $\mathrm{CV}$ combinations used in our study are relatively common across many languages, it would be of interest to investigate the effects of multilingualism on the planning processes involved in speech production such as those measured in the current study. In conclusion, the main purpose of the study reported in this paper was to compare movement-related oscillatory brain responses for verbal and button press tasks at two different complexity levels. Congruent with the findings from previous non-verbal motor studies, our findings have shown that both verbal and button press movements shown a significant desynchronization of beta oscillations during the movement preparation and holding phase and a re-synchronization upon movement termination. Importantly, both tasks also show a significant complexity effect in that the pre-movement phase for simple tasks is characterized by a transient partial synchronization. In contrast, our study revealed significant differences between the two tasks as well, especially in the role of bilateral vs. unilateral modulations and the specific role of the left hemisphere during the verbal task. Additionally, task complexity was found to affect the 
observed modulation patterns in both tasks differently. Therefore, our study suggests that while findings from the general motor control research can inform our understanding of speech motor control, significant differences exist between the two motor systems that caution against overgeneralization of underlying neural control processes. 


\section{Acknowledgements}

The authors would like to acknowledge Marc Lalancette for his assistance with collecting the brain imaging data and Vincci Tau and Nidhi Ravishanker for their contributions to data analysis. 


\section{References}

Alegre, M., Alvarez-Gerriko, I., Valencia, M., Iriarte, J., \& Artieda, J. (2008). Oscillatory changes related to the forced termination of a movement. Clinical Neurophysiology, 119(2), 290-300.

Bai, O., Mari, Z., Vorbach, S., \& Hallett, M. (2005). Asymmetric spatiotemporal patterns of event-related desynchronization preceding voluntary sequential finger movements: A highresolution EEG study. Clinical Neurophysiology, 116(5), 1213-1221.

Ballard, K. J., Robin, D. A., \& Folkins, J. W. (2003). An integrative model of speech motor control: A response to ziegler. Aphasiology, 17(1), 37-48.

Buzsaki, G. (2006). Rhythms of the brain. New York: Oxford University Press.

Chen, R., Gerloff, C., Hallett, M., \& Cohen, L. G. (1997). Involvement of the ipsilateral motor cortex in finger movements of different complexities. Annals of Neurology: Official Journal of the American Neurological Association and the Child Neurology Society, 41(2), 247-254.

Chen, R., Gerloff, C., Hallett, M., \& Cohen, L. G. (1997b). Involvement of the ipsilateral motor cortex in finger movements of different complexities. Annals of Neurology: Official Journal of the American Neurological Association and the Child Neurology Society, 41(2), 247-254.

Cheyne, D. O. (2013). MEG studies of sensorimotor rhythms: a review. Experimental Neurology, 245, 27-39.

Cheyne, D., Bakhtazad, L., \& Gaetz, W. (2006). Spatiotemporal mapping of cortical activity accompanying voluntary movements using an event-related beamforming approach. Human brain mapping, 27(3), 213-229. 
Cheyne, D., Bostan, A. C., Gaetz, W., \& Pang, E. W. (2007). Event-related beamforming: a robust method for presurgical functional mapping using MEG. Clinical Neurophysiology, 118(8), 1691-1704.

Cheyne, D. O., Ferrari, P., \& Cheyne, J. A.(2012). Intended Actions and Unexpected Outcomes: Automatic and Controlled Processing in a Rapid Motor Task. Front Hum Neuroscience, 6: 237

Christina, R. W., Fischman, M. G., Vercruyssen, M. J., \& Greg Anson, J. (1982). Simple reaction time as a function of response complexity: Memory drum theory revisited. Journal of Motor Behavior, 14(4), 301-321.

Dronkers NF (1996): A new brain region for coordinating speech articulation. Nature 384:159_ 161.

Eckert, M. A., Menon, V., Walczak, A., Ahlstrom, J., Denslow, S., Horwitz, A., \& Dubno, J. R. (2009). At the heart of the ventral attention system: the right anterior insula. Human brain mapping, 30(8), 2530-2541.

Fischman, M. G. (1984). Programming time as a function of number of movement parts and changes in movement direction. Journal of Motor Behavior, 16(4), 405-423.

Gaetz, W., Macdonald, M., Cheyne, D., \& Snead, O. C. (2010). Neuromagnetic imaging of movement-related cortical oscillations in children and adults: Age predicts post-movement beta rebound. NeuroImage, 51(2), 792-807. 
Gehrig, J., Wibral, M., Arnold, C., \& Kell, C. A. (2012). Setting up the speech production network: How oscillations contribute to lateralized information routing. Frontiers in Psychology, 3, 169.

Gould, I. C., Nobre, A. C., Wyart, V., \& Rushworth, M. F. (2012). Effects of decision variables and intraparietal stimulation on sensorimotor oscillatory activity in the human brain. Journal of Neuroscience, 32(40), 13805-13818.

Guenther, F. H. (2016). Neural control of speech. Mit Press.

Guthrie, D., \& Buchwald, J. S. (1991). Significance testing of difference potentials. Psychophysiology, 28(2), 240-244.

Hassan, M., Benquet, P., Biraben, A., Berrou, C., Dufor, O., \& Wendling, F. (2015). Dynamic reorganization of functional brain networks during picture naming. Cortex, 73, 276-288.

Heinrichs-Graham, E., \& Wilson, T. W. (2015). Coding complexity in the human motor circuit. Human Brain Mapping, 36(12), 5155-5167.

Houde, J. F., \& Nagarajan, S. S. (2011). Speech production as state feedback control. Frontiers in human neuroscience, 5,82 .

Isabella, S., Ferrari, P., Jobst, C., Cheyne, J. A., \& Cheyne, D. (2015). Complementary roles of cortical oscillations in automatic and controlled processing during rapid serial tasks. Neuroimage, 118, 268-281.

Jensen, O., Spaak, E. \& Zumer, J.M. (2014). Human brain oscillations: From physiological mechanisms to analysis and cognition. In Supek, S. \& Aine, Ch. J. 
(Ed.), Magnetoencephalography. from signals to dynamic cortical networks (pp. 359-403). Heidelberg: Springer.

Jobst, C., Ferrari, P., Isabella, S., \& Cheyne, D. (2018). BrainWave: a matlab toolbox for beamformer source analysis of MEG data. Frontiers in neuroscience, 12, 587.

Kaiser, J., Birbaumer, N., \& Lutzenberger, W. (2001). Event-related beta desynchronization indicates timing of response selection in a delayed-response paradigm in humans. Neuroscience Letters, 312(3), 149-152.

Kaiser, J., Ulrich, R., \& Lutzenberger, W. (2003). Dynamics of sensorimotor cortex activation to spatial sounds precueing ipsi-versus contralateral manual responses. Cognitive Brain Research, 17(3), 573-583.

Kent, R. D. \& Read, C. (1992). The acoustic analysis of speech. Singular Publishing Group San Diego.

Khanna, P., \& Carmena, J. M. (2015). Neural oscillations: Beta band activity across motor networks. Current Opinion in Neurobiology, 32, 60-67.

Kim, H., Lepage, C., Evans, A. C., Barkovich, A. J., \& Xu, D. (2015). NEOCIVET: Extraction of cortical surface and analysis of neonatal gyrification using a modified CIVET pipeline. Paper presented at the International Conference on Medical Image Computing and Computer-Assisted Intervention, 571-579.

Klapp, S. T. (2003). Reaction time analysis of two types of motor preparation for speech articulation: Action as a sequence of chunks. Journal of motor behavior, 35(2), 135-150. 
Kurz, M. J., Becker, K. M., Heinrichs-Graham, E., \& Wilson, T. W. (2014). Neurophysiological abnormalities in the sensorimotor cortices during the motor planning and movement execution stages of children with cerebral palsy. Developmental Medicine \& Child Neurology, 56(11), 1072-1077.

Lalancette, M., Quraan, M., \& Cheyne, D. (2011). Evaluation of multiple-sphere head models for MEG source localization. Physics in Medicine \& Biology, 56(17), 5621.

Little, S., Bonaiuto, J., Barnes, G., \& Bestmann, S. (2019). Human motor cortical beta bursts relate to movement planning and response errors. PLoS biology, 17(10), e3000479.

Loh, K. K., Procyk, E., Neveu, R., Lamberton, F., Hopkins, W. D., Petrides, M., \& Amiez, C. (2020). Cognitive control of orofacial motor and vocal responses in the ventrolateral and dorsomedial human frontal cortex. Proceedings of the National Academy of Sciences, 117(9), 4994-5005.

Maas, E. (2017). Speech and nonspeech: What are we talking about? International Journal of Speech-Language Pathology, 19(4), 345-359.

Manganotti, P., Gerloff, C., Toro, C., Katsuta, H., Sadato, N., Zhuang, P. a., . . Hallett, M. (1998). Task-related coherence and task-related spectral power changes during sequential finger movements. Electroencephalography and Clinical Neurophysiology/Electromyography and Motor Control, 109(1), 50-62.

Mary, A., Bourguignon, M., Wens, V., de Beeck, M. O., Leproult, R., De Tiège, X., \& Peigneux, P. (2015). Aging reduces experience-induced sensorimotor plasticity. A magnetoencephalographic study. Neuroimage, 104, 59-68. 
Mayka, M. A., Corcos, D. M., Leurgans, S. E., \& Vaillancourt, D. E. (2006). Three-dimensional locations and boundaries of motor and premotor cortices as defined by functional brain imaging: a meta-analysis. Neuroimage, 31(4), 1453-1474.

Munding, D., Dubarry, A., \& Alario, F. (2016). On the cortical dynamics of word production: A review of the MEG evidence. Language, Cognition and Neuroscience, 31(4), 441-462.

Nakagawa, K., Aokage, Y., Fukuri, T., Kawahara, Y., Hashizume, A., Kurisu, K., \& Yuge, L. (2011). Neuromagnetic beta oscillation changes during motor imagery and motor execution of skilled movements. Neuroreport, 22(5), 217-222.

Ng, T. H., Sowman, P. F., Brock, J., \& Johnson, B. W. (2013). Neuromagnetic brain activity associated with anticipatory postural adjustments for bimanual load lifting. Neuroimage, 66, 343-352.

Oldfield, R. C. (1971). The assessment and analysis of handedness: The edinburgh inventory. Neuropsychologia, 9(1), 97-113.

Park, H., Kim, J. S., \& Chung, C. K. (2013). Differential beta-band event-related desynchronization during categorical action sequence planning. PLoS One, 8(3).

Pastötter, B., Berchtold, F., \& Bäuml, K. T. (2012). Oscillatory correlates of controlled speedaccuracy tradeoff in a response-conflict task. Human Brain Mapping, 33(8), 1834-1849.

Piai, V., Roelofs, A., Rommers, J., Dahlslätt, K., \& Maris, E. (2015). Withholding planned speech is reflected in synchronized beta-band oscillations. Frontiers in Human Neuroscience, $9(\mathrm{OCT})$ 
Ricci, S., Tatti, E., Mehraram, R., Panday, P., \& Ghilardi, M. F. (2019). Beta band frequency differences between motor and frontal cortices in reaching movements. IEEE 16th International Conference on Rehabilitation Robotics (ICORR) (pp. 1254-1259).

Robinson, S. E., \& Vrba, J. (1998). Functional neuroimaging by synthetic aperture magnetometry SAM in: Biomag 2000. Paper presented at the 11th International Conference on Biomagnetism. Sendai, Japan, 1-4.

Sternberg, S., Monsell, S., Knoll, R. \& Wright, R. (1980). The latency and duration of rapid movement sequences: comparison of speech and typewriting. In Cole, R. (ed) Perception and production of fluent speech. Routhledge

Tallon-Baudry, C., Bertrand, O., Delpuech, C., \& Pernier, J. (1997). Oscillatory $\gamma$-band (30-70 hz) activity induced by a visual search task in humans. Journal of Neuroscience, 17(2), 722734.

Tan, H., Jenkinson, N., \& Brown, P. (2014). Dynamic neural correlates of motor error monitoring and adaptation during trial-to-trial learning. Journal of Neuroscience, 34(16), 5678-5688.

Tan, H., Wade, C., \& Brown, P. (2016). Post-movement beta activity in sensorimotor cortex indexes confidence in the estimations from internal models. Journal of Neuroscience, 36(5), $1516-1528$.

Tremblay, C., Champoux, F., Voss, P., Bacon, B. A., Lepore, F., \& Théoret, H. (2007). Speech and non-speech audio-visual illusions: A developmental study. PloS One, 2(8) 
Tzagarakis, C., Ince, N. F., Leuthold, A. C., \& Pellizzer, G. (2010). Beta-band activity during motor planning reflects response uncertainty. Journal of Neuroscience, 30(34), 1127011277.

Walsh, E., Kühn, S., Brass, M., Wenke, D., \& Haggard, P. (2010). EEG activations during intentional inhibition of voluntary action: An electrophysiological correlate of selfcontrol?. Neuropsychologia, 48(2), 619-626.

Wöstmann, M., Lim, S. J., \& Obleser, J. (2017). The human neural alpha response to speech is a proxy of attentional control. Cerebral cortex, 27(6), 3307-3317.

Ziegler, W., \& Ackermann, H. (2013b). Neuromotor speech impairment: It's all in the talking. Folia Phoniatrica Et Logopaedica, 65(2), 55-67. 


\section{Figures}

\section{Figure 1: Button Press and Verbal tasks}

A. Button press task with sample stimulus sequences, displayed for $800 \mathrm{~ms}$, followed by a variable inter-stimulus interval of $800-1800 \mathrm{~ms}$, then a green square as a 'go' cue for $500 \mathrm{~ms}$ to signal to subjects to enter their response in the response pad. The 'go' cue was followed by a fixation cross for $1600 \mathrm{~ms}$. B. The verbal task had the same sequence of events with sample stimuli displayed. Upon presentation of the green 'go' cue, subjects were required to utter their responses, recorded by a microphone.

\section{Figure 2: Source image for button press task}

Group average source images for simple and complex button press sequences revealed significant beta event-related desynchronization within the left $(x=-34, y=-17, z=51)$ and right $(x=36, y=-19, z=47)$ sensorimotor cortices. Images represent activity epoched to the first button press, thresholded from -15 to $15 \mathrm{nAm}$ relative to a pre-stimulus baseline time period of -0.4 to $0 \mathrm{~s}$.

\section{Figure 3: Time courses of beta activity during button press task $(n=15)$}

Time course of beta activity within the left (top) and right (bottom) sensorimotor cortex, epoched to stimulus onset (A), first button press (B), and last button press (C). Beta activity during simple sequences is represented by the green trace, while activity during complex sequences is represented by the red trace. Latencies of statistical differences between simple and complex 
traces $(p<0.05)$ are represented by the gray bar at the bottom of each plot. Above the traces is represented a schematic of the display timing.

\section{Figure 4: Source image for verbal task}

Group average source images for simple and complex verbal sequences revealed significant beta event-related desynchronization within the left $(x=-42, y=-6, z=30)$ and $\operatorname{right}(x=42, y=-6$, $z=35)$ sensorimotor cortices. Images represent activity epoched to the first plosive, thresholded -8 to 8 nAm relative to a pre-stimulus baseline time period of -0.4 to $0 \mathrm{~s}$.

\section{Figure 5: Time courses of beta activity during verbal task $(n=13)$}

Time course of beta activity within the left (top) and right (bottom) sensorimotor cortex, epoched to stimulus onset (A), first plosive (B), and last formant (C). Beta activity during simple sequences is represented by the green trace, while activity during complex sequences is represented by the red trace. Latencies of statistical differences between simple and complex traces $(p<0.05)$ are represented by the gray bar at the bottom of each plot. Above the traces is represented a schematic of the display timing. 
Figure 1:

A)
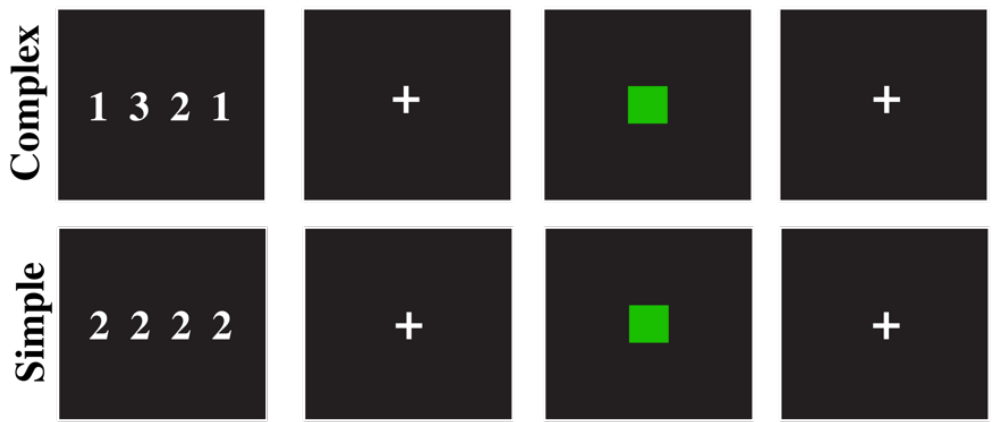

Stimulus

(800ms)

()

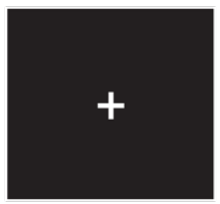

ISI

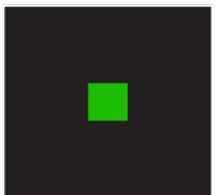

Cue ('Go')

(500ms)

800-1800ms)

()

$\square$

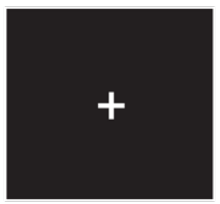

Fixation

(1600ms)

B)
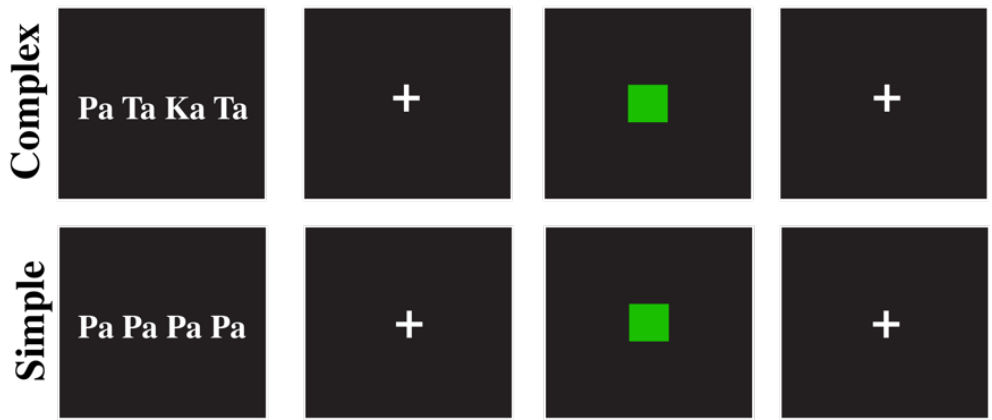

Stimulus

(800ms)

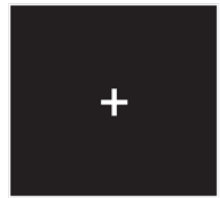

ISI

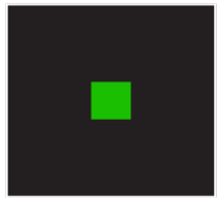

Cue ('Go')

(500ms)

()

(800-1600ms)

()

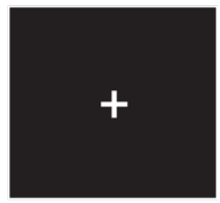

Fixation

(1600ms)

() 
Figure 2:

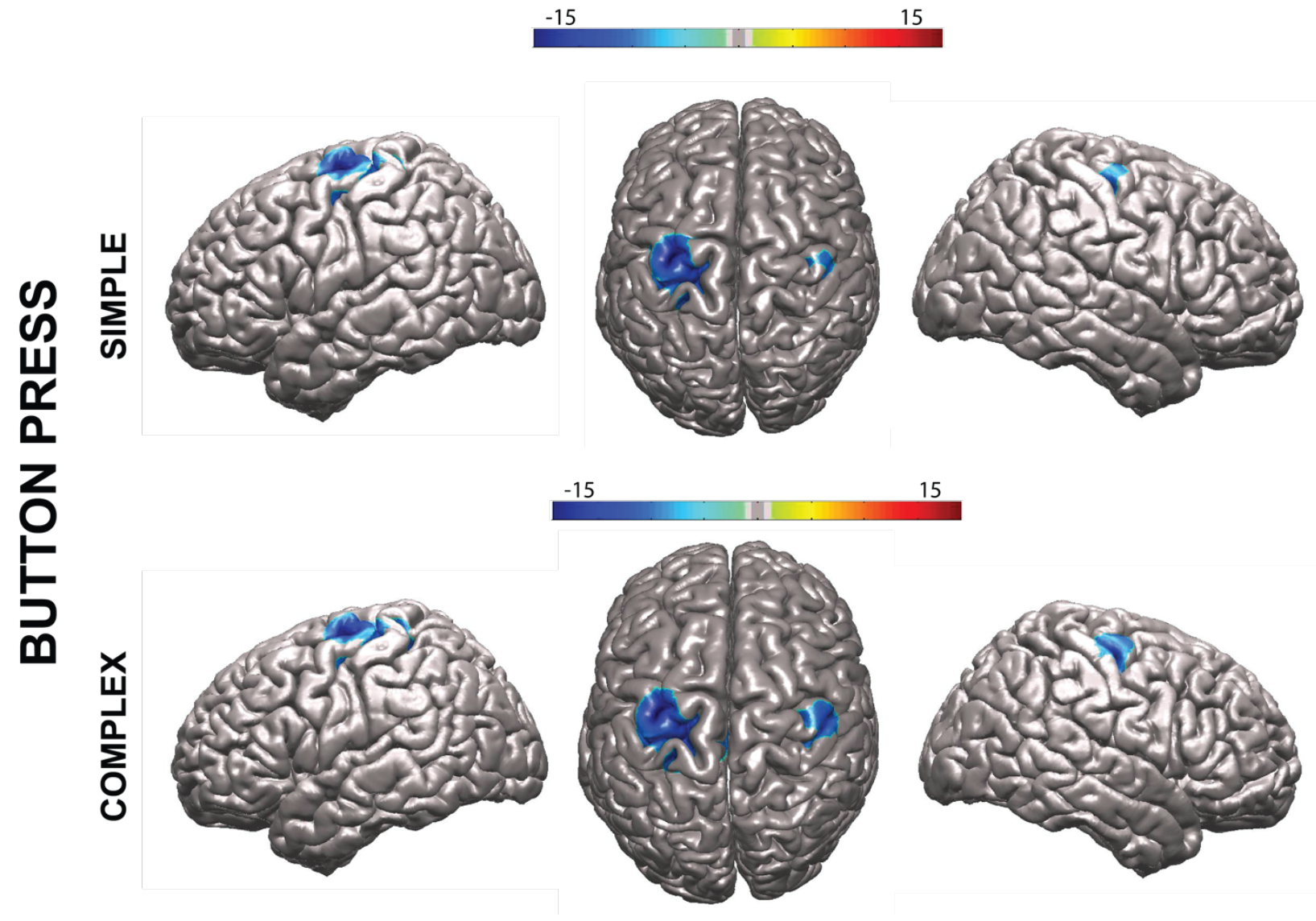


Figure 3:
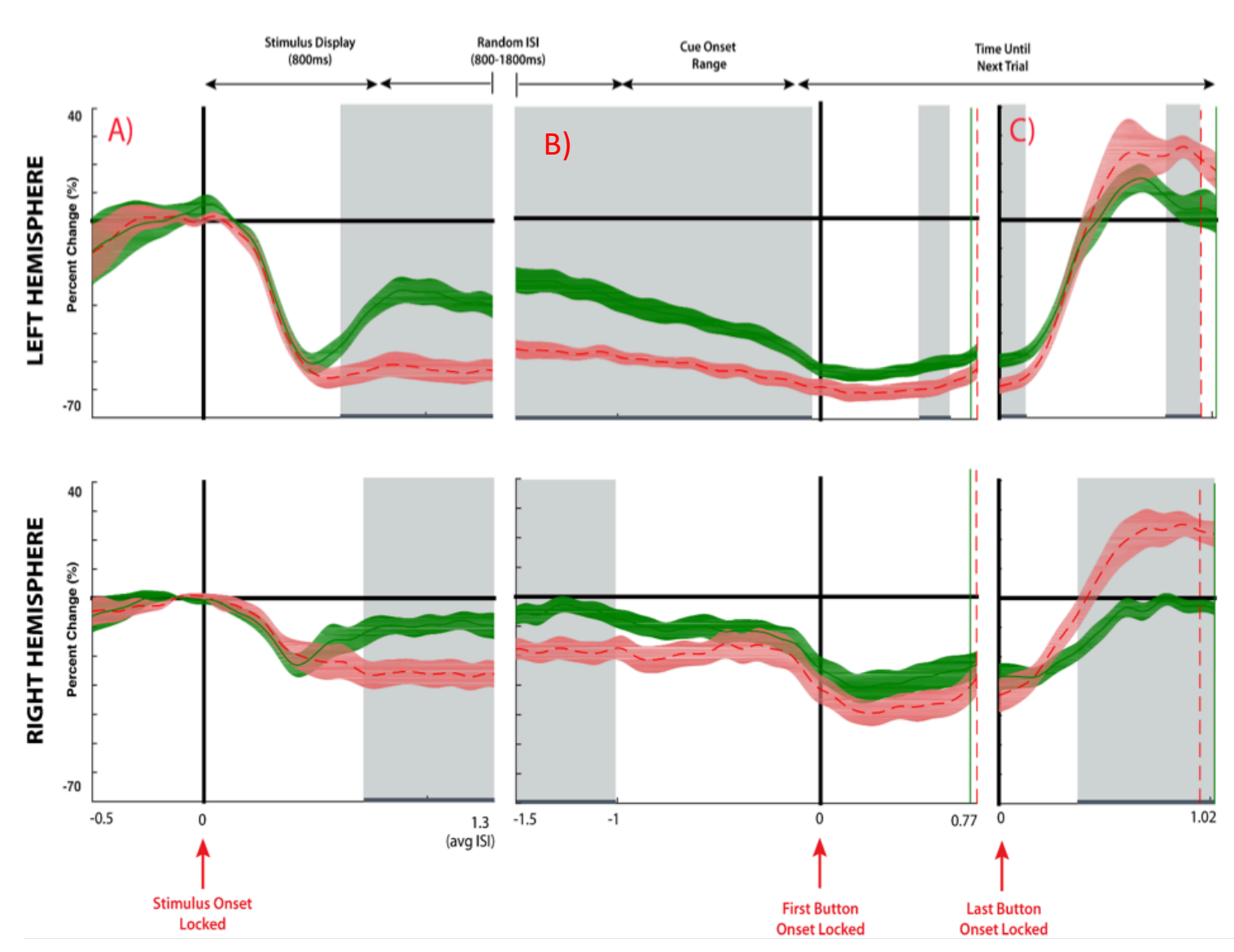

\begin{tabular}{|l|}
\hline \\
\hline Simple \\
Complex \\
\hline
\end{tabular} 
Figure 4:

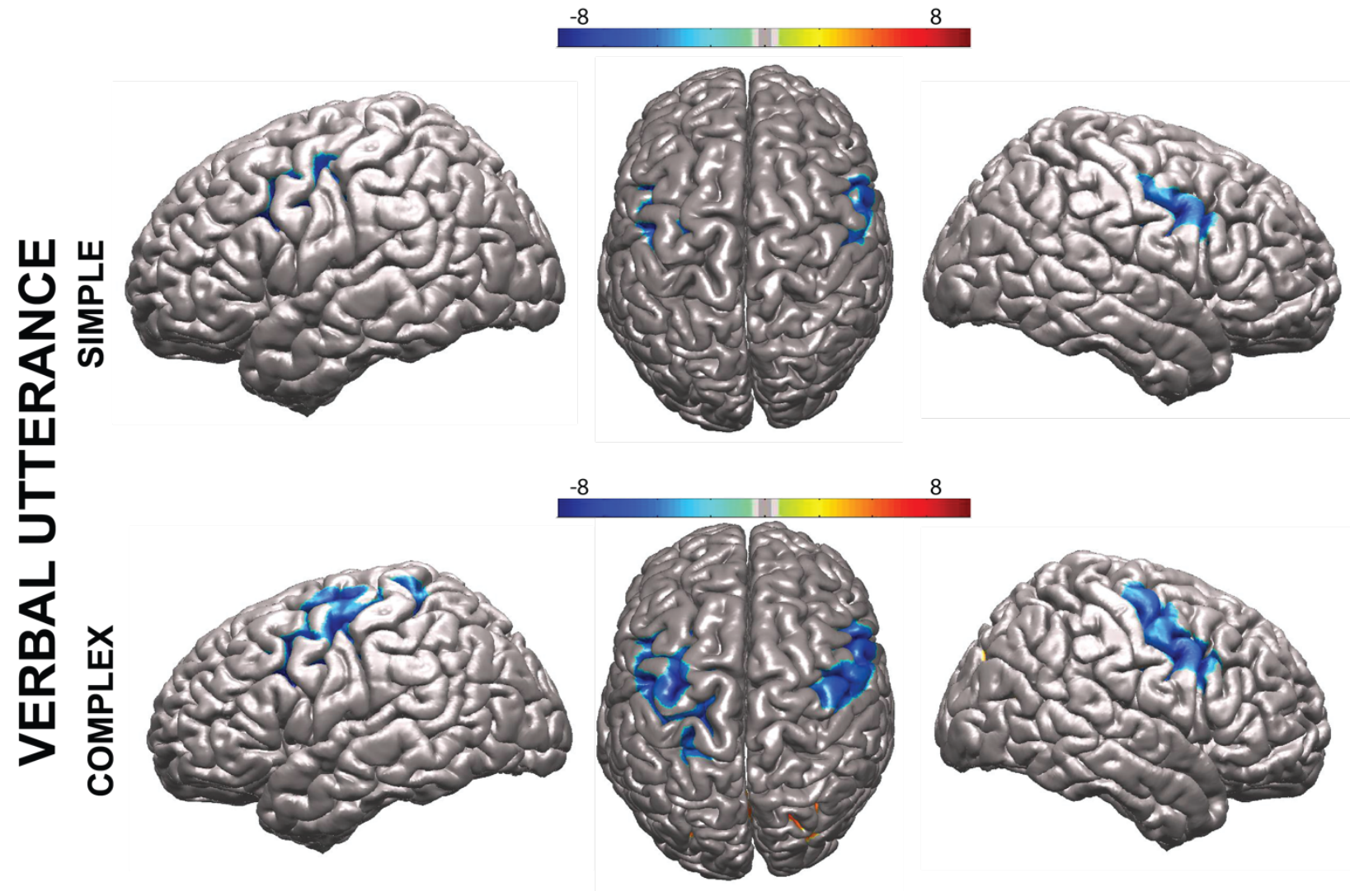


Figure 5:

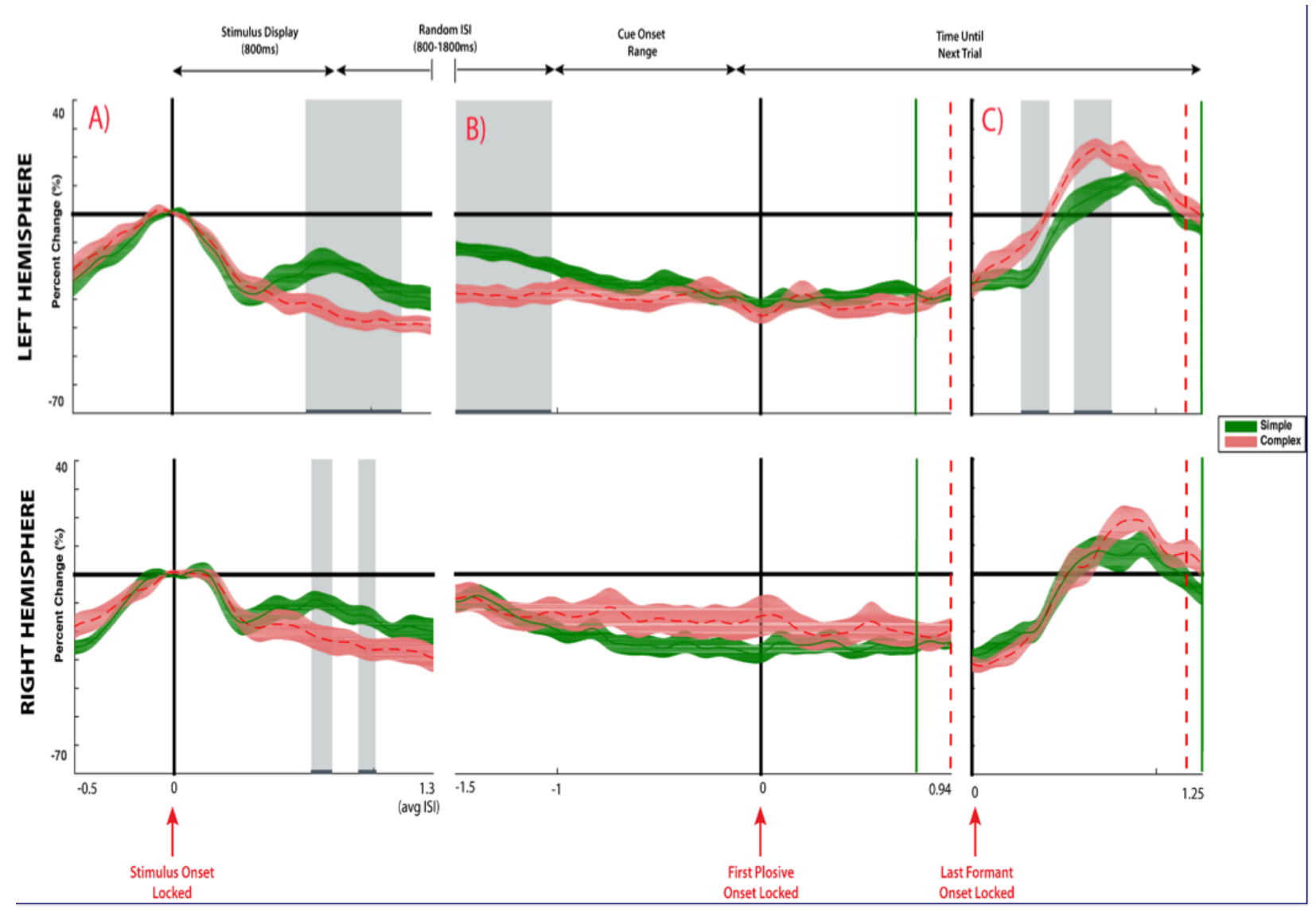


Tables 
Table 1: Mean and Standard Error (SE) of reaction times and response duration for button press and verbal tasks

\begin{tabular}{|l|c|c|c|c|c|}
\hline & \multicolumn{2}{|c|}{ SIMPLE } & \multicolumn{2}{c|}{ COMPLEX } & \\
\hline Button Press Task & Mean & SE & Mean & SE & $p$-value \\
\hline Reaction Time & 0.362 & 0.004 & 0.402 & 0.004 & $<0.001$ \\
\hline Response Duration & 0.736 & 0.084 & 0.768 & 0.085 & 0.19 \\
\hline Verbal Task & & & & & \\
\hline Reaction Time & 0.401 & 0.039 & 0.475 & 0.047 & $<0.0001$ \\
\hline Response Duration & 0.869 & 0.049 & 0.935 & 0.113 & 0.02 \\
\hline
\end{tabular}


Table 2: Mean and Standard Error (SE) of event-related desynchronization (ERD) latency and amplitude and event-related synchronization (ERS) for button press and verbal tasks

\begin{tabular}{|l|c|c|c|c|c|c|c|c|}
\hline & \multicolumn{4}{|c|}{ SIMPLE } & \multicolumn{3}{c|}{ COMPLEX } \\
\hline & \multicolumn{2}{|c|}{ LEFT } & \multicolumn{2}{c|}{ RIGHT } & \multicolumn{2}{c|}{ LEFT } & \multicolumn{2}{c|}{ RIGHT } \\
\hline Button Press Task & Mean & SE & Mean & SE & Mean & SE & Mean & SE \\
\hline ERD latency & 0.038 & 0.027 & 0.105 & 0.095 & 0.009 & 0.038 & 0.068 & 0.041 \\
\hline ERD amplitude & -66.0 & 5.5 & -29.0 & 4.2 & -68.5 & 5.4 & -38.5 & 3.6 \\
\hline ERS latency & 0.567 & 0.049 & 0.662 & 0.058 & 0.659 & 0.056 & 0.615 & 0.067 \\
\hline ERS amplitude & 73.6 & 5.8 & 38.8 & 3.9 & 108.3 & 8.6 & 76.8 & 8.1 \\
\hline Verbal Task & \multicolumn{7}{|c|}{} & \multicolumn{7}{|c|}{} & & & & \\
\hline ERD latency & 0.062 & 0.027 & 0.197 & 0.072 & 0.008 & 0.042 & 0.210 & 0.062 \\
\hline ERD amplitude & -66.0 & 5.5 & -29.0 & 4.2 & -68.0 & 5.4 & 38.5 & 3.6 \\
\hline ERS latency & 1.173 & 0.456 & 0.824 & 0.053 & 0.692 & 0.033 & 0.813 & 0.052 \\
\hline ERS amplitude & 48.1 & 5.3 & 52.3 & 4.5 & 67.2 & 5.3 & 57.2 & 6.0 \\
\hline
\end{tabular}


Table 3: Source localization of beta activity for Button Press and Verbal tasks

\begin{tabular}{|l|c|c|c|c|}
\hline & \multicolumn{2}{|c|}{ SIMPLE } & \multicolumn{2}{c|}{ COMPLEX } \\
\hline & LEFT & RIGHT & LEFT & RIGHT \\
\hline Button Press Task & $\mathbf{( x ,}, \mathbf{y}, \mathbf{z})$ & $\mathbf{( x ,}, \mathbf{y}, \mathbf{z})$ & $\mathbf{( x ,}, \mathbf{y}, \mathbf{z})$ & $\mathbf{( x , y , ~ z )}$ \\
\hline Stimulus & $-34,-21,43$ & $30,-21,43$ & $-34,-21,43$ & $34,-13,47$ \\
\hline First Button Press & $-34,-17,51$ & $34,-17,47$ & $-38,-17,51$ & $38,-21,47$ \\
\hline Last Button Press & $-34,-17,51$ & $38,-17,47$ & $-38,-17,43$ & $38,-21,43$ \\
\hline Verbal Task & & & & \\
\hline Stimulus & $-46,1,28$ & $46,1,28$ & $-42,5,27$ & $46,1,28$ \\
\hline First Plosive & $-42,-6,28$ & $46,1,28$ & $-42,-6,32$ & $38,-13,43$ \\
\hline Last Formant & $-38,-7,24$ & $46,-2,31$ & $-38,-7,24$ & $46,5,27$ \\
\hline
\end{tabular}

